\title{
Do left-handed players have a strategic advantage in table tennis?
}

\author{
Ivan Malagoli Lanzoni', Rocco Di Michele', Sandro Bartolomei' and Gabriele \\ Semprinil \\ IUniversity of Bologna, Italy \\ E-mail: ivan.malagoli@unibo.it
}

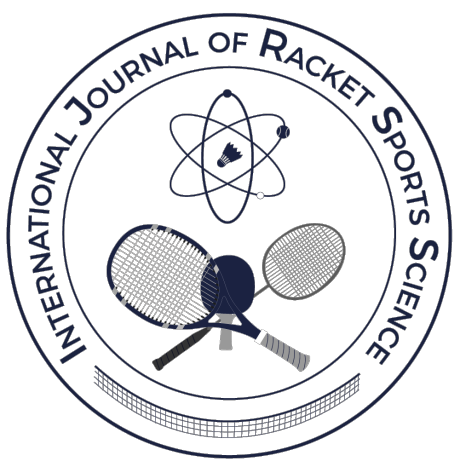

\begin{abstract}
The reasons for the overrepresentation of left-handed players (LHps) in some sports are widely discussed in the literature. In light of this debate, this study aimed to assess the associations between players' handedness and selected performance indicators in table tennis, where LHps represent $25 \%$ of top-level players. A notational analysis was conducted on 20 men's matches including any combination of players' handedness. Participants were in the first 150 positions of the ITTF world ranking at the moment the matches were played. The table area of ball bouncing after serving, and the shot type used by the receiving and subsequently the serving player, were recorded for 1515 rallies. Each half of the table was divided into six equal rectangular areas. There was a significant effect of players' handedness on percentage of ball bouncing in different areas. Specifically, LHps showed a greater capacity (or choice) to adjust the serve (in terms of areas of ball bouncing) than right-handed players (RHps) according to the opponent's handedness. Furthermore, LHps used offensive shots more frequently. In conclusion, both play strategy and characteristics such as higher offensiveness, together may contribute to the success of LHps in table tennis. These findings emphasise the need for a multifactorial approach in future research aiming to understand why LHps may be advantaged in different sports.
\end{abstract}

Keywords: Racket sports; Table tennis; Notational analysis; Handedness; Serve-return strategy

Cite this article as:

Malagoli Lanzoni, I., Di Michele, R., Bartolomei, S., \& Semprini, G. (2019). Do left-handed players have a strategic advantage in table tennis? International Journal of Racket Sports Science, 1(1), 61-69. 


\section{Introduction}

In many sports, and especially in those disciplines where the actions of players may directly affect the actions of their opponents (i.e., interactive sports), left-handed players (LHps) are overrepresented by up to $30 \%$ of competitors (Loffing, Hagemann, \& Strauss, 2009), leading to the supposition that left-handedness may be beneficial for achieving high competitive performance (Llaurens, Raymond, \& Faurie, 2009). An innate superiority linked to better neuropsychological predispositions (Bisiacchi, Ripoll, Stein, Simonet, \& Azemar, 1985; Dane \& Erzurumluoglu, 2003; Judge \& Stirling, 2003), and a negative frequency-dependent strategic advantage (Brooks, Bussiére, Jennions, \& Hunt, 2003; Raymond, Pontier, Dufour, \& Moller, 1996; Schorer, Loffing, Hagemann, \& Baker, 2012) are two well-known explanations of how LHps may be advantaged over right-handed (RH) players. Possible mechanisms contributing to an advantage for LHps were also postulated by researchers, who identified advantages linked to perceptual-cognitive skills (Loffing, Hagemann, Schorer, \& Baker, 2015) or to biomechanical factors (Solomito, Ferreir, \& Nissen, 2017).

The factors leading to the overrepresentation of LHps have been assessed in several interactive sports, including team sports such as basketball and soccer, and individual sports such as fencing, boxing and racket sports. In particular, previous findings in the tennis research area supports the view that LH tennis players have advantages of tactical or strategic nature (Hagemann, 2009; Loffing, Hagemann, \& Strauss, 2010). Indeed, many players are typically less accustomed to play against LHps than right-handed (RH) players, and might be not well prepared to counter effectively the shots made by these opponents. However, more recently, it was shown that LHps are overrepresented at amateur level but no more so among top-level players (Loffing, Hagemann, \& Strauss, 2012). A possible explanation is that professional players carefully analyze the matches played in the major tournaments and have a wide knowledge of their opponents' playing strategies. Therefore, at the highest competitive levels, left- handedness would seem to represent no more of an advantage for competitive performance.

Contrary to tennis, a high representation of LHps can still be observed among top-level table tennis players. Indeed, LHps represented $25 \%$ of the top 100 male players in the world ranking at Oct 2015 (data taken from the International Table Tennis Federation website, www.ittf.com). Since it is likely that the world's best table tennis players study their opponents with no less professionalism than tennis playing counterparts, LHps may have competitive advantages that cannot be nullified by the players' preparation.

The serve plays an important role for performance in racket sports (Aviles, Navia, Ruiz \& Martinez de Quel, 2019; Cui, Gómez, Gonçalves \& Sampaio, 2018; Katsikadelis, Pilianidis, \& Mantzouranis, 2013; Ma, Liu, Tan, \& Ma, 2013), and is certainly an aspect that may provide a key to understand how LHps are advantaged in table tennis. Loffing et al. (2009) assessed whether LHps could have an advantage related to the serve in professional tennis players. Those authors showed that the zone of the opponent's pitch in which the ball was sent, and the angle of lateral ball flight, was different between RHps and LHps, forcing the opponent to consider a different direction of the serve and to adjust the return stroke due to the different spin imposed on the ball. This finding led the authors to conclude that the serve is particularly relevant for the determination of a possible advantage of LHps over RH opponents. To our knowledge, no study has assessed the relationships between handedness and the serve bouncing/landing area in table tennis. In table tennis matches, the service imposes greater pressure on the receiver, creating favourable striking conditions for the next shot (Zhang, Liu, Hu, \& Liu, 2014). Moreover, the effectiveness of the serve and of the immediately subsequent shots has a great impact on the outcome of a rally in table tennis (Zhang et al., 2014). It may be thus hypothesised that, also in table tennis, the advantage of LHps may derive from characteristics of the serve and, consequently, from aspects related to the first shots of the rally.

In the recent years, scientific research has applied match and notational analysis to the most popular 
racket sports: badminton (Abdullahi \& Coetzee, 2017), tennis (Cui et al., 2018), and table tennis (Malagoli Lanzoni, Di Michele \& Merni, 2014; Fuchs et al., 2018). Using such an approach, this study aimed to assess the associations between the players' handedness and selected characteristics of the serve and the first two shots of the rally in top-150 table tennis matches.

\section{Methods}

\section{Data collection}

A total of 20 men's table tennis matches, played by 40 players (19 Europeans and 21 Asians) were examined. All players adopted an offensive playing style because they were not using a backhand chop stroke when playing far from the table and they did not use long-pimple rubbers, the typical rubbers used by defenders. The mean ( \pm SD) age, height and mass of the players were $26.3( \pm 5.3)$ years, $178.7( \pm 5.9) \mathrm{cm}$, and $70.7 \quad \pm 6.0) \quad \mathrm{kg}$ respectively (data taken from www.ittf.com). The matches sampled was based on a random selection of matches played between 2008 and 2014 by the top 150 players in the world in international events (Olympic Games, Individual and Team World Championship, World Cup, Pro Tour circuit, and ITTF world team classic). This kind of selection was done to include only one game for each player. All the matches were recorded from broadcasts of a free online TV channel, who agreed to the use of the video recordings for conducting the present study. The study was deemed exempt from ethical approval by the University of Bologna Bioethics Committee.

The handedness of players was established according to which hand was used to hold the racket (Peters \& Murphy, 1992). RHps $(\mathrm{n}=20)$ and LHps ( $=20$ ) were equally represented. Furthermore, 5 of the examined matches were played between two RHps, 5 between two LHps, and 10 between a RHp and a LHp. The matches were played to the best of five sets, finishing 3-2 $(n=4), 3-1(n=2)$, and 3-0 $(n=1)$, or to the best of seven sets, finishing 4-3 $(n=2), 4-2$ ( $n$ $=2), 4-1(n=7)$, and 4-0 $(n=2)$.

A table tennis coach with international coaching experience collected the examined indicators on a spreadsheet while watching the video of matches in slow motion with the software Kinovea (www.kinovea.org). The following indicators were recorded for each rally:

- $\quad$ Area of the table where the ball bounced after the player's serve (abbreviated as "Area"). According to a previous study (Malagoli Lanzoni et al., 2014), each half of the table was divided in six equal rectangular areas (see Figure 1). Three areas are in the front (closer to the net) part of the table, respectively numbered as 2 (front right), 3 (front center), and 4 (front left), and three areas are in the rear (closer to the player) part of the table, respectively numbered as 1 (rear right), 6 (rear center), and 5 (rear left)

- Type of shot used to hit the ball by a player when receiving the serve (defined as "S2", according to Zhang et al., 2013). Three shot types were considered according with literature (Malagoli et al., 2014): flick (attacking shot typically executed when the ball has bounced closed to the net), push (a neutral shot imparting a back-spin effect to the ball), and top (an attacking shot imparting a top-spin effect to the ball). These shot categories included the great majority of S2 shots. Rallies with a different S2 shot type, or rallies ended immediately after the serve without a response of the receiving player, were discarded from subsequent analyses.

- Type of shot used to hit the ball by the serving player when receiving the S2 shot (defined as "S3" according to Zhang, et al., 2014). Five shot types were considered: flick, push, top, block (a defensive shot performed in response to a top), and top counter top (a top, i.e. an offensive shot, performed against a top).

\section{Reliability}

For one randomly selected match, data of serve bouncing area, shots types used to return the service and to hit the subsequent ball were recorded by three national table tennis coaches. Furthermore, one of the coaches recorded the same match three times. Krippendorff's alpha (ranging between -1 and 1, where 1 indicates perfect agreement) was calculated to assess inter-and intra-operator reliability (Krippendorff, 2004). For the serve bouncing area and shot type, the inter-observer reliability alpha was equal to 0.94 and 
0.89 respectively and concerning the intra-observer reliability it was equal to 0.99 and 0.99 respectively.

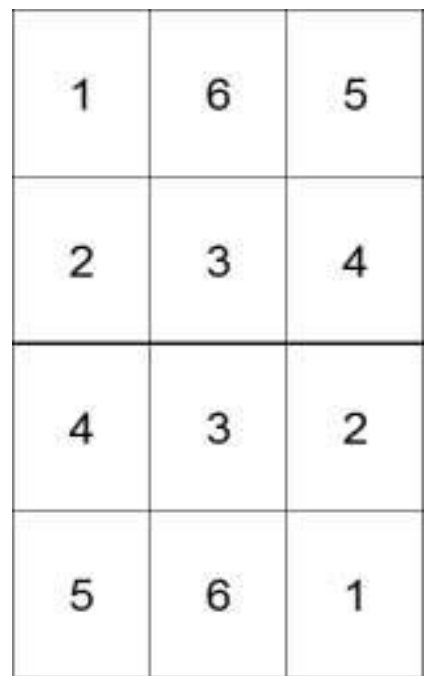

Figure 1. Six-area subdivision of the table. The thick line represents the net.

\section{Statistical Analysis}

All data are presented as the mean and standard deviations. For any player $(n=40)$, individual percentages of area of ball bouncing, S2 shot type and S3 shot type were calculated and used as the dependent variables. Six one-way ANOVAs with Tukey's post-hoc comparisons were used to assess the effect of players' handedness $(\mathrm{PH})$ on the mean percentages of areas of ball bouncing. Four $\mathrm{PH}$ categories were used in these analyses: both RHps (RR), both LHps (LL), differenthanded opponents with the RHp serving and the LHp receiving (RL), different-handed opponents with LHp serving and the RHp receiving (LR). Furthermore, twoway ANOVAs were used to assess the effects of the serving player handedness, opponent handedness, and their interaction, on the mean percentages of S2 (three ANOVAs) and S3 (five ANOVAs) shot types. In all ANOVAs, partial eta squared (\#2) were used as the effect size. The statistical analysis was carried out with the software R, version 3.2.3 (R Core Team, 2011). For all the analyses, the statistical significance was set at $\mathrm{p}<0.05$. For all the analyses (that is, those concerning the area of ball bouncing, the S2 shot type, and the S3 shot type), the alpha level was corrected dividing it by the number of statistical tests performed in that analysis.

\section{Results}

A total of 1515 rallies was examined for this analysis, with an average of 38 rallies per player. In each of the rallies, RHps executed the serve while standing close to area 5 (rear/left) of their own table side, whereas LHps executed the serve while standing close to area 1 (rear/right). The overall frequency distributions for, $\mathrm{PH}$, Area, S2, and S3, were, respectively, as follows:

- $\quad$ PH: RR: 344 (22.7 \%), RL: 397 (26.2 \%), LR: 412 (27.2 \%), LL: 362 (23.9\%);

- Areas: 1: 93 (6.1\%), 2: 199 (13.1\%), 3: 904 (59.7\%), 4: 204 (13.5\%), 5: 85 (5.6\%),

6: 30 (2.0\%);

- $\quad$ S2: flick: 300 (19.8 \%), push: 968 (63.9 \%), top: 247 (16.3\%);

- $\quad$ S3: block, 165 (10.9\%); flick, 141 (9.3\%); push, 295 (19.5\%); top, 794 (52.4\%);

top counter top, 120 (7.9\%).

Figure 2 shows the mean percentage values of area of ball bouncing for any players' handedness combination. For area 1, there was an effect of $\mathrm{PH}$ $\left(F_{3,36}=7.96, p<.01, \eta^{2}=0.40\right)$. Specifically, when both the players were left-handed, there was a higher percentage of balls sent into area 1 than in any other $\mathrm{PH}$ category. A significant effect for $\mathrm{PH}$ was also observed for area $2\left(\mathrm{~F}_{3,36}=5.36, \mathrm{p}<.01, \eta^{2}=0.31\right)$, with the condition of both right-handed players showing higher percentages of ball bouncing in area 2 than the conditions of both left-handed players and of righthanded serving players and left-handed receiving player. No effect of $\mathrm{PH}$ on mean percentage of ball bouncing was observed for areas $3\left(\mathrm{~F}_{3,36}=1.83, \mathrm{p}=.16\right)$, and $4\left(\mathrm{~F}_{3,36}=2.12, \mathrm{p}=.11\right)$. For area 5 , there was a significant effect of $\mathrm{PH}$ on percentage on ball bouncing in that area $\left(\mathrm{F}_{3,36}=4.43, \mathrm{p}<.01, \eta^{2}=0.27\right)$. Specifically, the post-hoc comparisons revealed a higher percentage in the condition of both right-handed players than in the condition of both left-handed players. Finally, no effect of $\mathrm{PH}$ on mean percentage of ball bouncing was observed for area $6(\mathrm{~F} 3,36=0.54, \mathrm{p}=.66)$. 


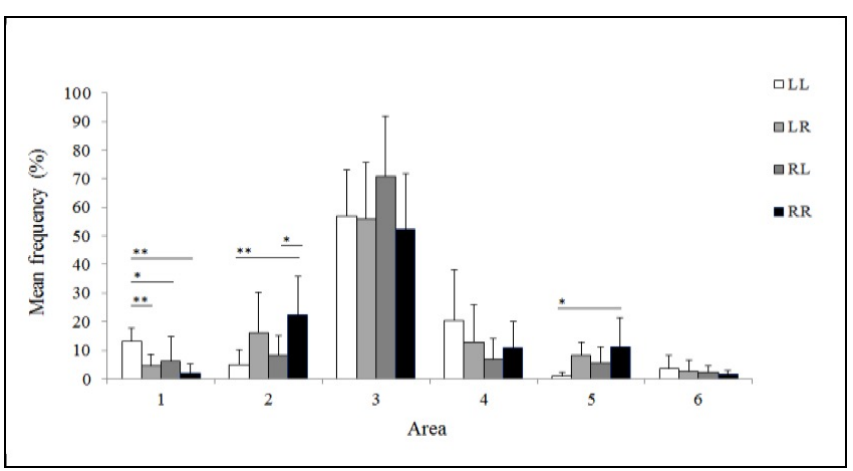

Figure 2. Mean and standard deviations (represented by vertical bars) frequencies for area of ball bouncing in the four examined handedness combinations.

$\mathrm{LL}=$ both LHp serving and receiving; $\mathrm{LR}=\mathrm{LHp}$ serving and $\mathrm{RHp}$ receiving; $\mathrm{RL}=\mathrm{RHp}$ serving and $\mathrm{LHp}$ receiving; $\mathrm{RR}=$ both RHp serving and receiving;

${ }^{*}=\mathrm{p}<0.05 ;{ }^{* *}=\mathrm{p}<0.01$
Tables 1 and 2 display the mean percentages of shot distribution for any combination of serving player's handedness and receiving player's handedness, for S2 and S3, respectively. For all S2 shot types, there was no significant effect of serving player's handedness, receiving player's handedness, and their interaction (all $\mathrm{p}>0.05)$. Similarly, no significant effect $(\mathrm{p}>0.05)$ was observed of serving and receiving player's handedness for S3 block, flick, top, and top counter top, nor for the main effect of serving player's handedness for the top $\left(\mathrm{F}_{1,36}=3.544, \quad \mathrm{p}=0.07, \eta 2=0.09\right)$, with left-handed players showing a higher mean percentage than righthanded players. Finally, for S3 push, there was a significant main effect of serving player's handedness $\left(\mathrm{F}_{1,36}=5.248, \mathrm{p}=0.03, \eta 2=0.13\right)$, with right-handed players showing a higher mean percentage of push shots than their left-handed counterparts.

Table 1.

Type of shot distribution of $S 2$ in relation to serving and receiving player's handednes.

\begin{tabular}{lccc}
\hline \multicolumn{3}{c}{ Players' handedness } & Shot type (mean $\% \pm$ SD) \\
\hline & Flick & Push & Top \\
LL & $22.7(14.8)$ & $58.2(15.4)$ & $19.1(7.7)$ \\
LR & $18.2(13.8)$ & $59.5(14.6)$ & $22.2(6.8)$ \\
RL & $18.8(6.7)$ & $62.4(19.0)$ & $18.8(13.9)$ \\
RR & $25.4(16.3)$ & $61.3(17.8)$ & $13.3(11.9)$ \\
\hline
\end{tabular}

$\mathrm{LL}=$ both LHp serving and receiving; $\mathrm{LR}=\mathrm{LHp}$ serving and $\mathrm{RHp}$ receiving; $\mathrm{RL}=\mathrm{RHp}$ serving and LHp receiving; $\mathrm{RR}=$ both RHp serving and receiving

Table 2.

Type of shot distribution of $S 3$ in relation to serving and receiving player's handedness.

\begin{tabular}{llcccc}
\hline \multicolumn{1}{c}{ Player handedness } & \multicolumn{5}{c}{ Shot type (mean \% \pm SD) } \\
\hline LL & Flick & Push & Top & Block & Top c top \\
LR & $9.2(6.7)$ & $15.8(9.2)$ & $56.9(9.1)$ & $12.3(5.2)$ & $5.7(3.3)$ \\
RL & $7.1(5.4)$ & $16.8(12.4)$ & $55.1(13.2)$ & $12.3(6.5)$ & $8.6(6.2)$ \\
RR & $9.7(8.3)$ & $23.3(11.1)$ & $47.8(12.4)$ & $10.4(11.2)$ & $8.7(10.7)$ \\
\hline
\end{tabular}

Top c top = top counter top.

$\mathrm{LL}=$ both $\mathrm{LHp}$ serving and receiving; $\mathrm{LR}=\mathrm{LHp}$ serving and $\mathrm{RHp}$ receiving; $\mathrm{RL}=\mathrm{RHp}$ serving and $\mathrm{LHp}$ receiving; $\mathrm{RR}=$ both RHp serving and receiving 


\section{Discussion and conclusions}

Previous studies suggest that left-handedness may be beneficial for achieving higher competitive performance (Llaurens et al., 2009) or enhances probability of success in interactive sports (Schorer et al., 2012). Therefore, the over representation of lefthanded athletes reflects their performance advantages in interactive sports (Loffing et al., 2015).

This study aimed to assess the associations between players' handedness and selected performance indicators in table tennis, where LHps represent $25 \%$ of top-level players.

In the present study, we assessed some aspects of the play strategy at the start of rallies in top 150 table tennis matches characterized by any different combinations of players' handedness. Notational analysis was chosen as the approach for data collection, since this method turned out to be effective to assess technical/tactical parameters in many sports including, first and foremost, racket sports (Abdullahi \& Coetzee, 2017; Fuchs et al., 2018; Hughes, 1998; Lees, 2003; Malagoli Lanzoni et al., 2014). The results showed an effect of players' handedness on area of ball bouncing and S3 shot type, suggesting that handedness can affect the play strategy.

The analysis of the effect of $\mathrm{PH}$ on mean percentage of ball bouncing in each area of the table (see Figure 2) provides a key to understanding whether and how lefthanded players can benefit of strategic advantages at the very start of rallies. This advantage could be linked to the possibility of playing high effective offensive shots such as top spin (Malagoli Lanzoni, Bartolomei, Di Michele, \& Fantozzi 2018), after the serve, by serving players. When both the players were $\mathrm{RH}$, a higher percentage of balls were sent towards areas 2 (close to the net on the forehand side) and 5 (close to the receiving player on the backhand side) than when both players were LL. Probably, forcing the opponent to stretch out and respond with a forehand was a deliberate strategy chosen by some players with the aim to make the opponent more vulnerable to a subsequent attack on the backhand corner. On the contrary, according to the above considerations, it's unlikely that any of the players aimed to send, intentionally, the ball towards area 5. Therefore, in matches between two RHps, most balls having reached area 5 were likely directed towards area 3 by the serving player, instead. Arguably, these balls bounced on area 5 (probably quite close to the centre of the table, i.e. at the boundary between areas 3 and 5), as a consequence of the sidespin effect of the ball and then to its trajectory. In other words, due to the side spin, the ball trajectory goes automatically toward backhand side, from area 3 to area 5 . A serve of this kind (known as forehand pendulum), interestingly, results in being not very easy to attack for the receiving player, despite the ball not bouncing close to the net. Indeed, differently from what would happen if the ball arrived in areas 1 and 6 , the receiver has to attack with a backhand top, a shot generally more difficult to execute than a forehand top. Moreover, in these serves, the ball tends to bounce for a second time near the table edge, resulting in increased hitting difficulty for the receiving player. Literature confirms that forehand top spin is the most used stroke used by top-level table tennis players (19.5\%) compared to top backhand (13.5\%), and it was more associated with winning outcomes (Malagoli Lanzoni et al., 2014).

When considering the matches between two LHp, given that the backhand and forehand sides are inverted for a LH receiver, the observed area pattern was consistent to that of matches between two RHp, with a higher percentage of balls reaching area 1 than in matches between two RHp, presumably for reasons similar to those noted above. Conversely, dissimilar patterns were observed between the two cases regarding different-handed players. On the one hand, when LHps served against RHps, the mean percentage of ball bouncing was almost similar to that of matches between two RHps, revealing no significant differences between LR and RR for any of the six examined areas (see Figure 2). On the other hand, when RHps served against LHps, the mean percentage of ball bouncing showed some differences when compared to that of LHps serving against LHps. In particular, when examining area 1 , a lower percentage of balls were sent to that area (close to the player on the backhand side) in the RL than in the LL condition. These results would seem to indicate that, to some extent, LHps are more 
capable than RHps to adjust their serving strategy to the opponent's handedness. At this playing level, it's reasonable to expect that any player knows very well what would be the optimal way to serve against any opponent, as in tennis (Loffing et al., 2009). Therefore, it is not straightforward to understand why RHps seem not to adapt their serving strategy, at least for what concerns the area of ball bouncing, when facing LHps opponents. Probably, many RHps believe a good solution is using in any case the serving technique they habitually train and master the best (the one optimal against RHps), despite the increased risk to be immediately attacked if the opponent is a LHp. In other words, these players do not consider it necessary to switch to a technique they are less confident with, only for the purpose to address the ball towards "less dangerous" areas. Irrespective of its reasons, the capacity (or choice) to adjust the serve (in terms of areas of ball bouncing) to the opponent's handedness, observed in LHp but not in RHp, may be regarded as a possible factor contributing to the success of LHp in top-level competitive table tennis.

An important effect of handedness was observed on the S3 shot type, even though there was not a main effect of serving player's handedness as revealed by two-way ANOVAs. In particular, irrespective of the opponent's handedness, left-handed players showed a lower percentage of push shots and a trend to a higher percentage of top shots as compared to right-handed players (Table 2). Since performing a top or a push can be considered, respectively, a more offensive or a more defensive approach to a similar situation faced by a player, these findings suggest that in this kind of match the LHp tend to generally adopt a more offensive strategy compared to RHp. This kind of behavior may be related to a possible higher aggressiveness of LHps, supposed to be a factor contributing to their success in competitive sport (Dane \& Sekertekin, 2005).

In summary, the present results show that the start of rallies in table tennis is influenced by the handedness of the two players. These findings, although limited to the set of selected indicators, provide clear information to explain how LHps may be advantaged in top-level table tennis. Our hypothesis that, under some aspects, the advantage of LHps could derive from serve characteristics, was supported by the results. Although limited to the area of ball bouncing as a descriptor of the serve effectiveness, it seems that LHps are more capable than RHps to optimally adapt the serving strategy to the opponent's handedness. This result corroborates a possible strategic advantage of LHps. Nevertheless, the analysis of the shot type at S2, and especially at S3, shows that RHps tend to make conservative choices, even if not forced to do so, while LHps tend to opt for more offensive choices. As in other sports, an innate higher aggressiveness of LHps may be a likely explanation for this kind of choice (Dane \& Sekertekin 2005). It may be concluded, therefore, that the advantage of left-handed players in top-level table tennis may have a multifactorial origin, showing links with both play strategy aspects and possibly innate characteristics such as aggressiveness.

Some limitations of the present study should be acknowledged. In particular, future perspectives should include the analysis of differences between male and female players, and between players of different performance levels (top-class, high-level, advanced, intermediate, beginner, etc.). Furthermore, the outcome of rallies may also be examined in relationship with other performance variables.

From a practical point of view, the present results suggest the need to improve technical-tactical skills through specific training and more attention needs to be given to the systematic introduction of specific exercises for the different handedness.

In conclusion, the present study showed that, in top150 table tennis players, the play strategy is influenced by the handedness of players. Indeed, left-handed players were more able than right-handed players to adapt the serving strategy to the opponent's handedness. Furthermore, left-handed players showed a more offensive play strategy. Therefore, the advantage of left-handed players in top-level table tennis may derive from both play strategy aspects and characteristics as higher aggressiveness. These findings add new insights to the debate on why left-handed players may be advantaged over right-handed players in interactive sports.

\section{References}


Abdullahi, Y., \& Coetzee, B. (2017). Notational singles match analysis of male badminton players who participated in the African badminton championships. International Journal of Performance Analysis in Sport, 17, $1-16$

Aviles, C., Navia, J. A., Ruiz, L. M., \& Martinez de Quel, O. (2019). Do expert tennis players actually demonstrate anticipatory behavior when returning a first serve under representative conditions? A systematic review including quality assessment and methodological recommendations. Psychology of Sport $\&$ Exercise, 43, 16-26.

Bisiacchi, P. S., Ripoll, H., Stein, J. F., Simonet, P., \& Azemar, G. (1985). Lefthandedness in fencers: an attentional advantage? Perceptual and Motor Skills, 100, 507-513.

Brooks, R., Bussiére, L. F., Jennions, M. D., \& Hunt, J. (2003). Sinister strategies succeed at the cricket World Cup. Proceedings of the Royal Society of London B: Biological Sciences, 271, S64-S66.

Cui, Y., Gómez,M. Á., Gonçalves, B., \& Sampaio, J. (2018). Performance profiles of professional female tennis players in grand slams. PLoS ONE, 13(7), e0200591..

Dane, S., \& Erzurumluoglu, A. (2003). Sex and handedness differences in eye-hand visual reaction times in handball players. International Journal of Neurosciences, 113, 923-929.

Dane, S., \& Sekertekin, M. A. (2005). Differences in handedness and scores of aggressiveness and interpersonal relations of soccer players. Perceptual and Motor Skills, 100, 743-746.

Fuchs, M., Liu, R., Malagoli Lanzoni, I., Munivrana, G., Straub, G., Tamaki, S., Yoshida, K., Zhang, H., \& Lames, M. (2018). Table Tennis Match Analysis: A Review. Journal of Sport Science, 36(23), 2653-2662.

Hagemann, N. (2009). The advantage of being left-handed in interactive sports. Attention, Perception \& Psychophysics, 71, 1641-1648.

Hughes, M. (1998). The application of notational analysis to racket sports. In: A. Lees,I. Maynard, M. Hughes, \& T. Reilly (Eds.), Science and Racket Sports II (pp. 211-220) London, UK: E \& FN Spon.
Judge, J., \& Stirling, J. (2003). Fine motor skill performance in left-and right- handers: evidence of an advantage for left-handers. Laterality, 8, 297-306.

Katsikadelis, M., Pilianidis, T., \& Mantzouranis, N. (2013). The interaction between serves and match winning in table tennis players in the London 2012 Olympic Games. International Journal of Table Tennis Sciences, 8, 77-79.

Krippendorff, K. (2004). Content's analysis: An introduction to its methodology. Thousand Oaks, CA: Sage.

Lees, A. (2003). Science and the major racket sports: a review. Journal of Sports Science, 21, 707-732.

Llaurens, V., Raymond, M., \& Faurie, C. (2009). Why are some people left-handed? An evolutionary perspective. Proceedings of the Royal Society of London B: Biological Sciences, 364, 881-894.

Loffing, F., Hagemann, N., \& Strauss, B. (2009). The serve in professional men's tennis: effects of players' handedness, International Journal of Performance Analysis in Sport, 9, 255-274.

Loffing, F., Hagemann, N., \& Strauss, B. (2010). Automated processes in tennis: do left-handed players benefit from the tactical preferences of their opponents? Journal of Sports Science, 28, 435-443.

Loffing, F., Hagemann, N., \& Strauss, B. (2012). Left-handedness in professional and amateur tennis. Plos One,7(11), e49325.

Loffing, F., Hagemann, N., Schorer, J., \& Baker, J. (2015). Skilled players' and novice's difficulty anticipating left- vs. right-handed opponents' action intentions varies across different points in time. Human Movement Science, 40, 410-421.

Ma, S. M., Liu, C. C., Tan, Y., \& Ma, S. C. (2013). Winning matches in grand slam men's singles: An analysis of player performance-related variables from 1991 to 2008. Journal of Sports Science, 31, 1147-55.

Malagoli Lanzoni, I., Bartolomei, S., Di Michele, R., \& Fantozzi, S. (2018). A kinematic comparison between long-line and cross-court top spin forehand in competitive table tennis players. Journal of Sports Science, 36, 2637-2643.

Malagoli Lanzoni, I., Di Michele, R., \& Merni, F. (2014). A notational analysis of shot characteristics in 
top-level table tennis players. European Journal of Sport Science, 14, 309-317.

Peters, M., \& Murphy, K. (1992). Cluster analysis reveals at least three, and possibly five distinct handedness groups. Neuropsychologia, 30(4), 373-380.

Raymond, M., Pontier, D., Dufour, A. B., \& Moller, A. P. (1996). Frequency dependent maintenance of left handedness in humans. Proceedings of the Royal Society of London B: Biological Sciences, 263, 1627-1633.

R Core Team (2011). R: A language and environment for statistical computing. Vienna, Austria. R Foundation for Statistical Computing.

Schorer, J., Loffing, F., Hagemann, N., \& Baker, J. (2012). Human handedness in interactive situations: negative perceptual frequency effects can be reversed! Journal of Sports Science, 30, 507-513.

Solomito, M. J., Ferreir, J. V., \& Nissen, C. V. (2017). Biomechanical differences between left and right-handed baseball pitchers. Sports Biomechanics, 16, 143-151.

Zhang, H., Liu, W., Hu, J. J., \& Liu, R. Z. (2014). Evaluation of elite table tennis players' technique effectiveness. Journal of Sports Science, 32, 70-77. 